\title{
(6) \\ Causal analysis of H1N1pdm09 influenza infection risk in a household cohort
} OPEN ACCESS

\author{
Yohann Mansiaux, ${ }^{1,2}$ Nicolas Salez, ${ }^{3}$ Nathanael Lapidus, ${ }^{1,2,4}$ Michel Setbon, ${ }^{5}$ \\ Laurent Andreoletti, ${ }^{6,7}$ Marianne Leruez-Ville, ${ }^{8}$ Simon Cauchemez, ${ }^{9}$ \\ Marie-Lise Gougeon, ${ }^{10}$ Frédéric Vély, 11,12,13,14 Michael Schwarzinger, $15,16,17$ \\ Laurent Abel, ${ }^{18,19}$ Rosemary Markovic Delabre, ${ }^{1,2}$ Antoine Flahault, ${ }^{20,21}$ \\ Xavier de Lamballerie, ${ }^{3}$ Fabrice Carrat ${ }^{1,2,4}$
}

- Additional material is published online only. To view please visit the journal online (http://dx.doi.org/10.1136/jech2014-204678).

For numbered affiliations see end of article.

\section{Correspondence to} Yohann Mansiaux, Equipe 2, UMRS-1136, Faculté de Médecine Saint Antoine, 27 Rue Chaligny, 75571 Paris, Cedex 12, France; yohann.mansiaux@upmc.fr

Received 16 July 2014 Revised 10 October 2014 Accepted 15 October 2014 Published Online First 21 November 2014

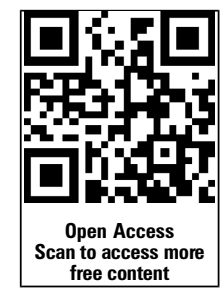

CrossMark

To cite: Mansiaux $Y$, Salez N, Lapidus N, et al. J Epidemiol Community Health 2015;69:272-277.

\section{ABSTRACT}

Background Obtaining a comprehensive quantitative figure of the determinants of influenza infection will help identify priority targets for future influenza mitigation interventions. We developed an original causal model integrating highly diverse factors and their dependencies, to identify the most critical determinants of pandemic influenza infection (H1N1pdm09) during the 2010-2011 influenza season.

Methods We used data from 601 households (1450 participants) included in a dedicated cohort. Structural equations were used to model direct and indirect relationships between infection and risk perception, compliance with preventive behaviours, social contacts, indoor and outdoor environment, sociodemographic factors and pre-epidemic host susceptibility. Standardised estimates $\left(\beta_{\text {std }}\right)$ were used to assess the strength of associations (ranging from -1 for a completely negative association to 1 for a completely positive association). Results Host susceptibility to H1N1pdm09 and compliance with preventive behaviours were the only two factors directly associated with the infection risk $\left(\beta_{\mathrm{std}}=0.31\right.$ and $\left.\beta_{\mathrm{std}}=-0.21\right)$. Compliance with preventive behaviours was influenced by risk perception and preventive measures perception $\left(\beta_{\text {std }}=0.14\right.$ and $\left.\beta_{\text {std }}=0.27\right)$. The number and duration of social contacts were not associated with H1N1pdm09 infection. Conclusions Our findings suggest that influenza vaccination in addition to public health communication campaigns focusing on personal preventive measures should be prioritised as potentially efficient interventions to mitigate influenza epidemics.

\section{INTRODUCTION}

Influenza infection results from a complex interplay between biological characteristics of the virus and host, individual and collective behaviours, social interactions and environmental factors. Numerous studies have investigated risk factors for pandemic influenza infection (H1N1pdm09): factors as diverse as individual characteristics (eg, young age, ${ }^{1}$ female gender, ${ }^{1}$ chronic comorbidity ${ }^{2}$ and low preepidemic antibody titre $^{3}$ ), indoor and outdoor environments (eg, number of participants in the household ${ }^{4}$ and residence in an urban area ${ }^{3}$ ), contacts with infected individuals, ${ }^{15}$ and use of preventive measures such as handwashing ${ }^{6}$ have been found to be determinants of H1N1pdm09 infection. However, no study has simultaneously explored these factors to provide a comprehensive figure of the determinants of influenza infection. The transmission mechanisms of the virus are therefore unclear and a question remains of how this information can be used by policymakers to effectively plan future mitigation strategies.

Conceptually, the determinants condition two unobserved components causally linked to infection, namely exposure to the virus and host susceptibility. For example, the number of contacts or use of preventive measures is a factor related to exposure, while haemagglutination antibody (HAI) titres, acquired naturally or after influenza vaccination, are related to host susceptibility. Other factors such as risk perception, beliefs and behaviours may act on both exposure and susceptibility, for example, by increasing social distance or vaccination.

In this study, a causal model with structural equations was used to explicitly model direct and indirect relationships between H1N1pdm09 infection and perception of infection risk, compliance with preventive behaviours, social contacts, indoor and outdoor environment, sociodemographic factors and pre-epidemic host susceptibility. Through the integration of various risk factors known to play a role in H1N1pdm09 infection, we aimed to identify the most critical determinants and thus help prioritise clear targets for future public health interventions.

\section{METHODS}

\section{Data source}

We used data from the CoPanFlu-France cohort, which was created to study H1N1pdm09 infection risk. The cohort comprises 601 households (1450 participants) randomly selected between December 2009 and July 2010 and actively monitored for influenza-like illness (ILI) during two consecutive influenza seasons (2010-2011 and 2011-2012). When ILI was reported (fever $\geq 37.8^{\circ} \mathrm{C}$ and cough and/or sore throat, with no other known cause ${ }^{7}$ ), nasal swabs were collected from every household member by a study nurse and screened for respiratory viruses. Serum samples were collected for pre-seasonpost-season HAI titration. Questionnaires exploring medical history, risk perception, use of preventive measures and duration and location of social contacts were administered at inclusion. Study protocol, data collection and representativeness of the study households' details can be found elsewhere. ${ }^{8}$ Study protocol was approved 
by the research ethics committee 'Comité de Protection des Personnes Ile-de-France 1'. All participants gave written informed consent. H1N1pdm09 infection was defined by either positive H1N1pdm09 RT-PCR or a positive H1N1pdm09 RespiFinder assay on a nasal swab, or seroconversion (fourfold increase in the HAI titre). Among the 1450 individuals included in the study, 132 withdrew participation before the end of the 2010-2011 season (17 for familial and/or health reasons and 64 due to the study burden; 47 participants were lost to follow-up, and 4 died). This analysis therefore focused on 1318 participants (559 households). Infection status was unavailable for 197 individuals $(14.9 \%)$ because at least one serological sample was missing. These participants were nevertheless included in the analysis of other covariates.

\section{Hypothesised structural model}

We postulated that influenza infection results from two unobserved phenomena: virus exposure and host susceptibility (figure 1). Their simultaneous impact on H1N1pdm09 infection risk was modelled using a structural equation model (SEM, see online supplementary file). SEM allows one to estimate direct and indirect relationships between observed or latent variables. Latent variables are unobserved variables, estimated from several observed variables, called indicators.

We modelled exposure to H1N1pdm09 with seven latent variables and one observed variable: cumulative incidence of ILI in the relevant French administrative regions. Host susceptibility to H1N1pdm09 infection was modelled with a single indicator: the pre-epidemic HAI titre (see online supplementary file and Lapidus $e t a l^{9}$ for additional information on covariates).

\section{Contact network}

Seven continuous indicators were used for this latent variable: daily number and duration of contacts with individuals $<15$ years, between 15 and 50 years, and $>50$ years and daily time spent in public transport. A contact was a verbal exchange (at least three words) between the participant and another individual or a physical contact. Duration and location of contacts, as well as the age of the individuals, were collected annually. For each participant, we used all the contact data collected as close to and/or during the 2010-2011 epidemic season (20 December 2010-20 February 2011 (eg, if a participant had multiple preepidemic or postepidemic contact data, we considered the information collected as close to the start and the end of the influenza season, respectively). The duration and number of individual contacts were averaged over the repeated contact data and summed according to the contact's age and were logtransformed (a value of 0.01 was imputed for participants reporting a zero summed duration or number of contacts). Reported durations of a given contact were additional, that is, a 20 min contact with three individuals corresponded to a summed contact duration of $60 \mathrm{~min}$.

\section{Perception of infection risk and of preventive measures}

Risk perception and opinions on preventive measures have been shown to impact individual preventive behaviours. ${ }^{10}{ }^{11}$ Two latent variables were used to model participants' perception and beliefs about H1N1pdm09 influenza based on responses to a dedicated questionnaire (see figure 1 for covariates used). Specifically, all household participants $>15$ years indicated whether they 'totally agree'/"partly agree'/"partly disagree'/ 'totally disagree' to statements such as 'prevention highly depends on behaviours' and 'H1N1pdm influenza is most often fatal'. For this analysis, the answers were dichotomised ('agree'/ 'disagree'). For household participants $<15$ years, we imputed for each missing covariate the most frequent answer by adults in the same household.

\section{Compliance with preventive behaviours}

Compliance with preventive measures has been shown to reduce H1N1pdm09 infection risk. ${ }^{6}{ }^{12}$ Three binary indicators were used: 'always/often washes hands after coughing/sneezing', 'always/often covers mouth while coughing or sneezing' and 'daily frequency of handwashing (with soap or hand sanitizer) $\geq 5$, dichotomised around the median daily frequency of handwashing.

\section{Indoor characteristics}

Six categorical indicators were used to describe living room and bedroom heating (electric/gas/other), average temperatures (dichotomised around their medians) and presence of air humidifiers. The possible impact of relative humidity on influenza aerosol transmission has been reported, and high indoor heating levels, especially during winter months, may lead to continuous circulation of dry air, creating an environment favourable to infectious viral particles persistence. ${ }^{13}$

\section{Neighbourhood socioeconomic status}

Geocoding of participants' addresses allowed us to assign each participant a geographic unit defined by INSEE (Institut National de la Statistique et des Etudes Economiques), IRIS zones corresponding to statistical block groups of about 2000 inhabitants. ${ }^{14}$ Three continuous indicators, provided by INSEE, were used to describe neighbourhood socioeconomic status: 'employment rate (\%) among 15-65 years', 'proportion (\%) of inhabitants $>15$ years without a diploma' and 'mean annual income ( $\mathrm{k} €)^{\prime}$ (log-transformed).

\section{Urban environment}

Three binary indicators were used for this latent variable: 'lives in a urban area' (defined by a 6 -item variable provided by INSEE), 'presence of agricultural land near habitation' and 'presence of livestock near habitation'.

\section{Host susceptibility to H1N1pdm09 infection}

We used a pre-epidemic HAI titre (log-transformed) to measure host susceptibility to H1N1pdm09 infection. An HAI titre is used by the European Medicines Agency (EMA) and the US Food and Drug Administration (FDA) to evaluate vaccine protection. ${ }^{15} 16$

\section{Additional observed covariates}

We postulated that participants' age class $(<15,15-50$, $>50$ years) would influence contact network, compliance with preventive behaviours, and differential susceptibility. Factors associated with high pre-epidemic HAI titres, such as influenza vaccination and ILI history during the previous season, were also investigated. Potential associations between age class, gender and vaccination were tested (relationships between observed covariates and observed/latent variables are shown in the online supplementary file).

ILI incidence in the relevant French administrative regions was used as a proxy of exposure. Using data from the French Sentinel Network, we estimated for each French administrative region the cumulative weekly incidence of ILI (defined as the abrupt onset of fever $>39^{\circ} \mathrm{C}$ with myalgia and respiratory signs) cases per 100000 population for the 2009-2010 (7 September 2009-27 December 2009) and 2010-2011 (20 December 


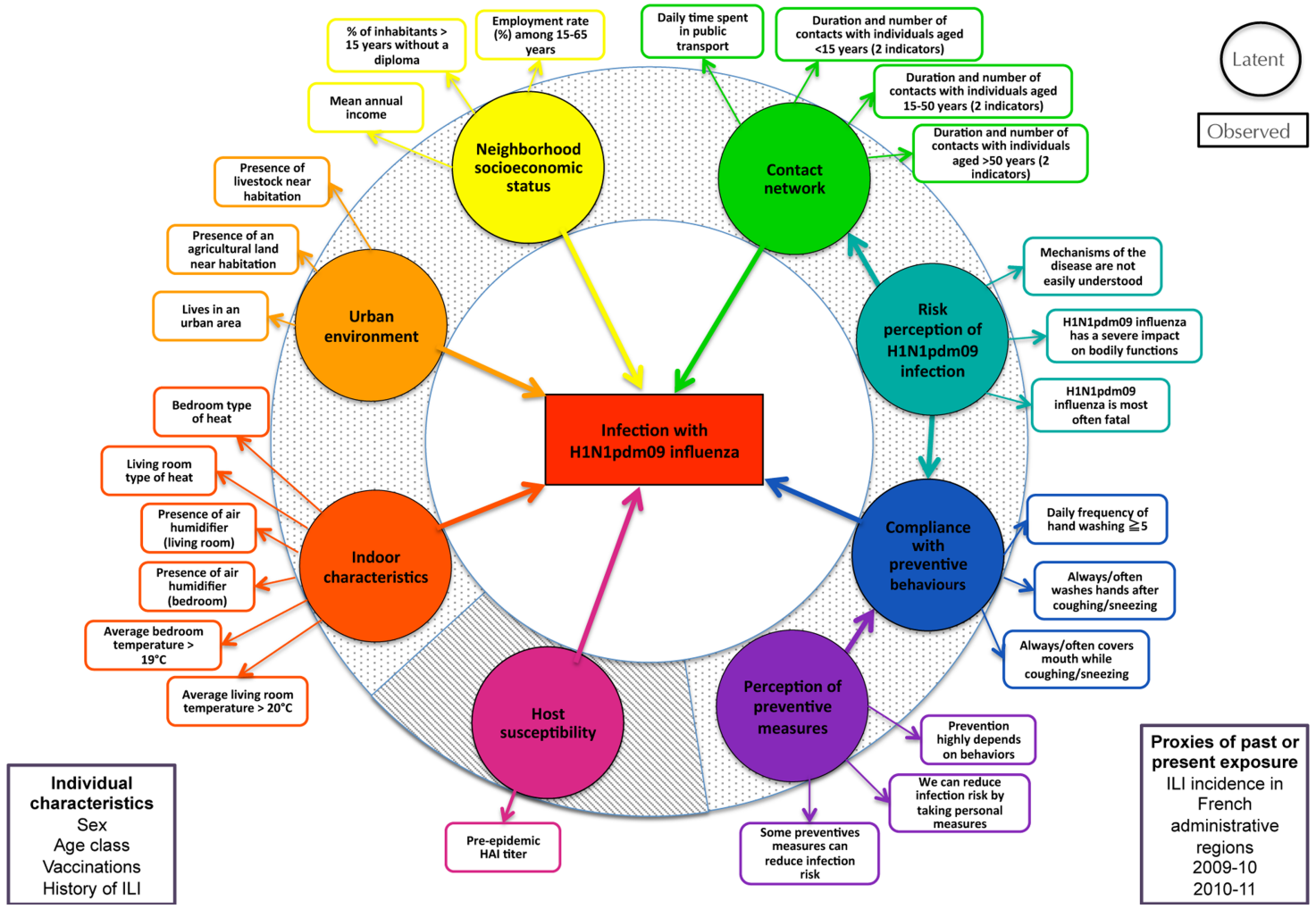

Figure 1 Hypothesised structural equation model. Ellipses: latent variables; boxes: observed variables. Dotted background: latent variables related to exposure to H1N1pdm09. Striped background: latent variable related to susceptibility to H1N1pdm09 infection. For clarity, relationships involving the additional observed variables are not shown.

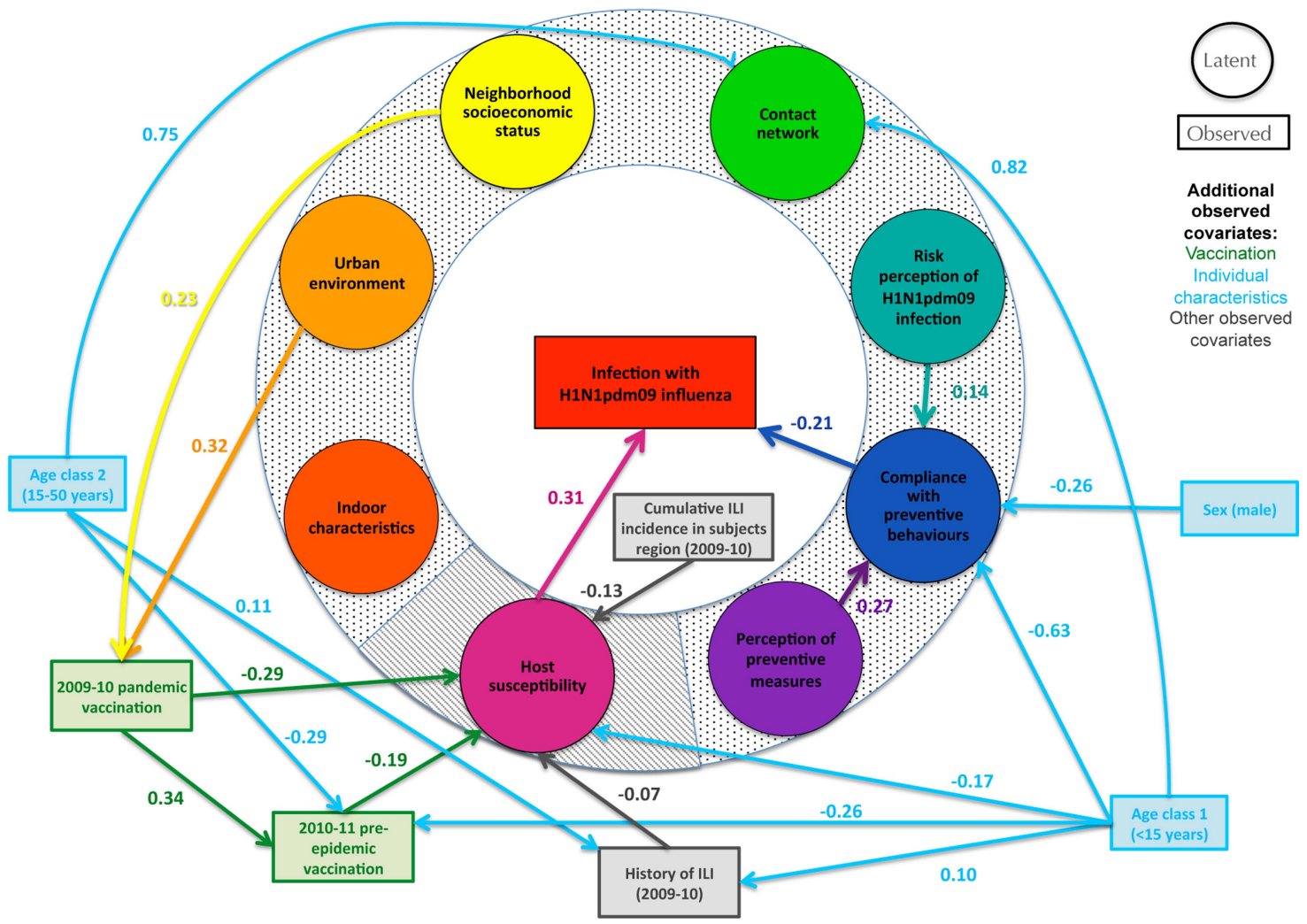

Figure 2 Final structural equation model. All coefficients have $p$ values $<0.05$. Ellipses: latent variables; boxes: observed variables. Dotted background: latent variables related to exposure to $\mathrm{H} 1 \mathrm{~N} 1 \mathrm{pdm} 09$ virus. Striped background: latent variable related to susceptibility to H1N1pdm09 infection. 
2010-20 February 2011) seasons. ${ }^{17}$ While the cumulative incidence of ILI per region in 2010-2011 was expected to be associated with the 2010-2011 infection, we assumed that the 2009-2010 cumulative ILI incidence would affect H1N1pdm09 host susceptibility.

\section{Model estimation}

Mplus 7 software was used to estimate the parameters of the structural model while accounting for within-household correlations. For easier interpretation of the coefficients, we report standardised estimates $\left(\beta_{\text {std }}\right)$ with $\mathrm{p}$ values $<0.05$. Standardised estimates (ranging from -1 for a completely negative association to 1 for a completely positive association) can be interpreted with reference to other estimates and the relative strength of associations can be compared. Goodness of fit was assessed with two criteria: the comparative fit index (CFI) and the root mean square error of approximation (RMSEA). The CFI ranges from 0 to 1 , with values $>0.90$ corresponding to an acceptable fit, while an RMSEA value $<0.08$ is recommended. ${ }^{18}$ To improve goodness of fit, new relationships can be added following 'modification indices' (approximations of model fit improvement when new structural equations are added) or non-significant and inconsistent relationships can be removed.

\section{RESULTS}

Data

Among the 1318 participants included in the study, 46.4\% were male (612 participants). Two hundred and fifty-seven participants were $<15$ years $(19.5 \%$; 24 infections; 9.3\%), 545 were aged $15-50$ years $(41.3 \%$; 36 infected; $6.6 \%)$ and 516 were $>50$ years $(39.2 \% ; 29$ infected; 5.6\%). One hundred and twenty-seven participants received the 2010-2011 seasonal influenza vaccine (9.6\%), compared to 180 participants vaccinated against H1N1pdm09 for the 2009-2010 season (13.7\%). Ninety-six participants (7.3\%) had ILI during the 2009-2010 season. Infection status was determined for 1121 participants (89 infections, 7.9\% of the participants with a known infectious status), which were used to estimate relationships with H1N1pdm09 infection.

\section{Structural-equation model of H1N1pdm09 infection}

The final SEM model is shown in figure 2 (coefficients of the relationships between latent variables and their indicators as well as a summary of the added and removed relationships are provided in the online supplementary file). H1N1pdm09 host susceptibility and compliance with preventive behaviours were the only two factors directly associated with H1N1pdm09 infection $\left(\beta_{\text {std }}=0.31, \mathrm{p}\right.$ value $<0.001$ and $\beta_{\text {std }}=-0.21, \mathrm{p}$ value $\left.=0.011\right)$. Compliance with preventive behaviours was positively influenced by the latent variables 'risk perception of H1N1pdm09 infection' and 'perception of preventive measures' $\left(\beta_{\text {std }}=0.14, p\right.$ value $=0.029$ and $\beta_{\text {std }}=0.27, p$ value $=0.001$ ). Participants $<15$ years and those aged 15-50 years were more exposed through contacts than were participants $>50$ years $\left(\beta_{\text {std }}=0.82\right.$ and $\beta_{\text {std }}=0.75, \mathrm{p}$ values $<0.001$ ). These two younger populations were less frequently vaccinated during the 2010-2011 season $\left(\beta_{\text {std }}=-0.26, \mathrm{p}\right.$ value $=0.018$ and $\beta_{\text {std }}=-0.29, \mathrm{p}$ value $\left.<0.001\right)$ and were more likely to have had ILI during the previous season $\left(\beta_{\text {std }}=0.10, \mathrm{p}\right.$ value $=0.022$ and $\beta_{\text {std }}=0.11, \mathrm{p}$ value $\left.=0.017\right)$. Participants $<15$ years were less compliant with preventive behaviours than those belonging to the older age groups $\left(\beta_{\text {std }}=-0.63\right.$, $\mathrm{p}$ value $<0.001)$, and males were less compliant than females $\left(\beta_{\text {std }}=-0.26, \mathrm{p}\right.$ value $\left.<0.001\right)$. Participants $<15$ years were the least susceptible to H1N1pdm09 ( $\beta_{\text {std }}=-0.17$, p value $\left.<0.001\right)$.
Vaccination during both the 2009-2010 and 2010-2011 seasons, ILI during the 2009-2010 season, and a high cumulative ILI incidence in the participants' administrative regions (20092010 season) were associated with lower host susceptibility $\left(\beta_{\text {std }}=-0.29, \mathrm{p}\right.$ value $=0.007 ; \beta_{\text {std }}=-0.19, \mathrm{p}$ value $<0.001 ; \beta_{\text {std }}=$ $-0.07, \mathrm{p}$ value $=0.033$ and $\beta_{\mathrm{std}}=-0.13$, $\mathrm{p}$ value $=0.018$, respectively). Individuals vaccinated during the 2009-2010 season were more likely to be vaccinated during the following season $\left(\beta_{\text {std }}=0.34, p\right.$ value $\left.=0.008\right)$. Individuals living in an urban area with a 'high' neighbourhood socioeconomic status (eg, a high mean income in the IRIS zone) were more likely to be vaccinated against pandemic influenza for the 2009-2010 season $\left(\beta_{\text {std }}=0.32, \mathrm{p}\right.$ value $=0.006$ and $\beta_{\text {std }}=0.23, \mathrm{p}$ value $\left.=0.010\right)$. Model goodness-of-fit indices were satisfactory (RMSEA $=0.023$, $\mathrm{CFI}=0.943)$.

\section{DISCUSSION}

Using structural equation modelling, we were able to obtain a coherent quantitative picture of the complex mechanisms determining H1N1pdm09 infection during the 2010-2011 influenza season. To the best of our knowledge, our study is the first to simultaneously integrate factors as diverse as contacts, risk perceptions, preventive behaviours and environmental and socioeconomic factors. Risk factor studies since 2009 have mainly been based on regression analysis, which may fail to capture the complex mechanisms underlying infection by estimating only direct, independent associations between covariates and H1N1pdm09 infection. Use of a structural-equation model allowed us to explicitly model direct and indirect relationships between highly diverse factors and their respective impacts on infection. Our results are particularly relevant to informing public health strategies; they can be used to determine which dimensions should be priority targets of public health communication campaigns.

Host susceptibility, measured here with a pre-epidemic HAI titre, was the major factor explaining infection. ${ }^{3}{ }^{19}$ Influenza vaccination, ILI history during the first H1N1pdm09 season, and the level of community incidence, were the factors associated with host susceptibility. ${ }^{20}$

Our results also suggest that compliance with preventive behaviours played an important role in influenza infection. ${ }^{12}$ Compliance with preventive behaviours was related to a positive perception of the impact of such measures and to the risk perception of infection. ${ }^{21}$ Lower preventive behaviour compliance among men compared with women has previously been observed. ${ }^{11}$ Urban area of residence and neighbourhood socioeconomic status had no direct influence on infection, but both factors positively influenced the probability of 2009-2010 vaccination. Urban residence has been reported to have a positive influence on seasonal influenza vaccination. ${ }^{22}$ No relationship between vaccination and high neighbourhood socioeconomic status has previously been reported, but a high level of education has been linked to pandemic vaccine uptake, ${ }^{23}$ as has living in a household where the head of the family has a high professional status. ${ }^{24}$

The infection rate during the second season was $7.9 \%$ in the CoPanFlu cohort, a figure consistent with estimated seroconversion rates of $6.2 \%$ and $6.8 \%$ reported by Chen $e t$ al ${ }^{25}$ during the second and third H1N1pdm09 waves in Singapore. Participants $<15$ years were the least susceptible in our study (see online supplementary file). This may be explained by a higher rate of H1N1pdm09 infection during the 2009-2010 season; $10.3 \%$ of young participants in the CoPanFlu cohort reported ILI during this period. A similar relationship between a 
high rate of infection among children during the 2009-2010 season and lower susceptibility in 2010-2011 has been reported. ${ }^{20}$

No relationship was found to exist between exposure through the contact network and H1N1pdm09 infection. Several studies have nevertheless linked school closures or holiday periods to a reduced infection rate, ${ }^{26}$ implying a major influence of contacts with children on the individual infection risk. However, deeper analyses (see online supplementary file) did not suggest a higher level of exposure among infected participants. There are two possible explanations for this finding. First, contact information was not necessarily collected at the time when influenza was present in the community, especially during winter months. We used a methodology similar to that of the POLYMOD study and obtained highly consistent findings regarding assortativity and intensity of contacts according to age. ${ }^{27}$ This type of measure is now often used to explore social contact influence on the spread of infectious diseases. ${ }^{28-31}$ Contacts were also stable across calendar seasons (see online supplementary file). However, we cannot formally exclude that the pattern of contacts observed might not be representative of contacts with infected participants; contact patterns/behaviours of susceptible individuals with infectious participants may be modified in this context. Second, the daily number and duration of contacts were high in all age groups. Consequently, the likelihood of contacts with one or several infectious participants over the 2-month influenza season might be so high that transmissibility would not be influenced by the number of occasions, but rather by host characteristics.

Another limitation of our study was related to the modelling of the host susceptibility, which was only based on the preepidemic HAI titre. Integration of alternative correlates of protection such as T-cell responses, ${ }^{32}$ or of genetic factors, ${ }^{33}$ may provide a more robust picture of host susceptibility.

Among the 1450 participants included, 132 were not followed for the considered season and another 197 had a missing infectious status. Comparison of these 329 participants with the 1121 individuals with a known infectious status showed that the participants in the first group were older (46 years on average vs 38 years), were living in households with a lower number of participants (2.9 participants on average vs 3.1) and were more likely to be vaccinated during the 2009-2010 season (vaccination rate $18 \%$ vs $11 \%$ ). These last two characteristics were associated with the participant's age. No differences were observed for sex, 2010-2011 seasonal vaccination, ILI in 20092010, and pre-epidemic HAI titres. Thereby, considering the low differences between the two groups for most of these characteristics and that the influence of the participant's age on several factors under study was taken into account in our analyses, we can assume that the absence of infectious status information from these 329 participants is unlikely to bias the general conclusions of our work.

Compared with previous studies, our work has two major strengths. First, households were randomly sampled through a probabilistic procedure and selected households were shown to be representative of the general population (see Lapidus et $a l^{8}$ for more details). Second, participants were prospectively followed through an active weekly surveillance system over the two influenza periods. Both limit the risk of selection and information bias.

To the best of our knowledge, our study is also the first to simultaneously integrate such a variety of influenza risk factors through a causal approach. Structural equation models, however, cannot unequivocally prove causal relationships: acceptable indices of goodness of fit merely indicate that postulated relationships are supported by the data. Other models may provide equivalent or better performance, and covariates potentially involved in the phenomenon under study might have been omitted. $^{34}$

\section{CONCLUSION}

Structural equation modelling should be considered as a valuable approach to steer public health policies. While our results rely on data collected from the 2010 to 2011 influenza season, we feel the risk factors identified would be relevant in future epidemic and pandemic contexts. The nature of the influenza virus requires the production of a new annual vaccine to lower individual susceptibility. Risk perception and compliance with preventive behaviours, though modifiable, are unlikely to change over time without targeted intervention. We suggest that communication campaigns focus on infection risk and preventive measures efficacy, specifically targeting men and children. Additionally, we find that measures based on social distancing should not be a primary public health mitigation strategy, but warrant further investigation.

\section{What is already known on this subject}

- Since 2009, numerous studies have investigated risk factors for pandemic influenza infection, reporting factors as diverse as individual characteristics, indoor and outdoor environments, contacts with infected individuals and use of preventive measures.

- Questions remain about the respective roles of the reported determinants in the mechanism of influenza virus transmission.

- Knowledge on how this information can be used to guide policymakers to implement effective mitigation strategies is lacking.

\section{What this study adds}

- Using a novel methodological approach, we were able to rank the relative impacts of all the determinants of infection, thereby determining priority targets for public health communication campaigns. Pre-epidemic antibody titres, compliance with preventive behaviours, as well as risk perception, were the only factors directly or indirectly associated with the infection risk.

- Social behaviour does not seem to be a major determinant of infection.

- On the basis of our findings, we believe it is likely that public health communication campaigns stressing the efficacy of preventive measures and the risks associated with pandemic influenza infection would be effective to mitigate the influenza burden.

\section{Author affiliations}

'INSERM, UMR_S 1136, Institut Pierre Louis d'Epidémiologie et de Santé Publique, Paris, France

2UPMC Univ Paris 06, UMR_S 1136, Institut Pierre Louis d'Epidémiologie et de Santé Publique, Sorbonne Universités, Paris, France 
${ }^{3}$ IRD French Institute of Research for Development, EHESP French School of Public Health, UMR_D 190 "Emergence des Pathologies Virales", Aix Marseille Univ, Marseille, France

${ }^{4}$ Public Health Unit, Saint-Antoine Hospital, Paris, France

${ }^{5}$ IRD French Institute of Research for Development, EHESP French School of Public Health, EPV UMR_D 190 "Emergence des Pathologies Virales", CNRS-Aix Marseille Université, Marseille, France

${ }^{6}$ Laboratoire de Virologie médicale et moléculaire Hôpital Robert Debré, CHU Reims, Reims, France

${ }^{7}$ Faculté de Médecine, EA 4684, Reims, France

${ }^{8}$ Laboratory of Virology, Hospital Necker-.Enfants-malades, Assistance PubliqueHôpitaux de Paris APHP—University Paris Descartes, Sorbonne Paris Cité, Paris, France

${ }^{9}$ Mathematical Modelling of Infectious Diseases Unit, Institut Pasteur, Paris, France

${ }^{10}$ Antiviral Immunity, Biotherapy and Vaccine Unit, Institut Pasteur, Paris, France

${ }^{11}$ Centre d'Immunologie de Marseille-Luminy, INSERM, U1104, Marseille, France

${ }^{12}$ CNRS, UMR7280, Marseille, France

${ }^{13}$ Aix Marseille Université, UM2, Marseille, France

${ }^{14}$ Service d'Immunologie, Assistance Publique-Hôpitaux de Marseille, Hôpital de la Conception, Marseille, France

${ }^{15}$ IAME, UMR 1137, INSERM, Paris, France

${ }^{16}$ IAME, UMR 1137, Sorbonne Paris Cité, Univ Paris Diderot, Paris, France

${ }^{17}$ Translational Health Economics Network, Paris, France

${ }^{18}$ Laboratory of Human Genetics of Infectious Diseases, Necker Branch, Institut National de la Santé et de la Recherche Médicale U1163, Paris, France ${ }^{19}$ Imagine Institute, Paris Descartes University, Sorbonne Paris Cité, Paris, France

${ }^{20}$ Centre Virchow-Villermé, Descartes, Université Sorbonne Paris Cité, Paris, France

${ }^{21}$ Global Health Institute, University of Geneva, Geneva, Switzerland

Acknowledgements The authors thank David Young for a critical review of the manuscript.

Contributors NS, NL, MS, LA, ML-V, SC, M-LG, FV, MSc, LAb, RMD, AF and XdL critical revision of the manuscript. NL, MS, RMD and FC participated in the acquisition of data. NS and XdL performed serological analyses. NL, AF and FC drafted the protocol. NL, NS, MS, RMD, M-LG, FV, ML-V, LA, SC, LAb, MSC, AF, $\mathrm{XdL}$ and $\mathrm{FC}$ contributed to conception of the study. YM performed the experiments and analysed the data. YM and FC were involved in writing the manuscript.

Funding This study was supported by the Institut de Microbiologie et Maladies Infectieuses (IMMI-AVIESAN), the French Ministry of Health and Assistance Publique Hôpitaux de Paris-PHRC 2010 \#AOM10199, the French Ministry of Research and the Institut de Recherche en Sante Publique (IReSP_TGIR 2009).

Competing interests $\mathrm{FC}$ reported not having any shares or paid employment with pharmaceutical companies; he received honoraria from Novartis, GlaxoSmithKline, Astra Zeneca and Boiron and received travel support to attend scientific meetings from Novartis.

Patient consent Obtained.

Ethics approval Comité de Protection des Personnes lle-de-France 1.

Provenance and peer review Not commissioned; externally peer reviewed.

Open Access This is an Open Access article distributed in accordance with the Creative Commons Attribution Non Commercial (CC BY-NC 4.0) license, which permits others to distribute, remix, adapt, build upon this work non-commercially, and license their derivative works on different terms, provided the original work is properly cited and the use is non-commercial. See: http://creativecommons.org/ licenses/by-nc/4.0/

\section{REFERENCES}

1 France AM, Jackson M, Schrag S, et al. Household transmission of 2009 influenza A (H1N1) virus after a school-based outbreak in New York City, April-May 2009. $J$ Infect Dis 2010;201:984-92.

2 Sugimoto JD, Borse NN, Ta ML, et al. The effect of age on transmission of 2009 pandemic influenza A (H1N1) in a camp and associated households. Epidemiology 2011;22:180-7.

3 Chao D-Y, Cheng K-F, Li T-C, et al. Factors associated with infection by 2009 pandemic H1N1 influenza virus during different phases of the epidemic. Int I Infect Dis 2011;15:e695-701.

4 Cauchemez S, Donnelly CA, Reed C, et al. Household transmission of 2009 pandemic influenza A (H1N1) virus in the United States. N Engl J Med 2009;361:2619-27.

5 Lau LLH, Nishiura H, Kelly $H$, et al. Household transmission of 2009 pandemic influenza $A$ (H1N1): a systematic review and meta-analysis. Epidemiology 2012;23:531-42

6 Simmerman JM, Suntarattiwong P, Levy J, et al. Findings from a household randomized controlled trial of hand washing and face masks to reduce influenza transmission in Bangkok, Thailand. Influenza Other Respi Viruses 2011;5:256-67.
7 CDC. Flu activity and surveillance: reports and surveillance methods in the United States. http://www.cdc.gov/flu/weekly/fluactivitysurv.htm (accessed 31 Mar 2014).

8 Lapidus N, De Lamballerie $X$, Salez N, et al. Integrative study of pandemic A/H1N1 influenza infections: design and methods of the CoPanFlu-France cohort. BMC Public Health 2012:12:417.

9 Lapidus N, de Lamballerie X, Salez N, et al. Factors associated with post-seasonal serological titer and risk factors for infection with the pandemic $\mathrm{A} / \mathrm{H} 1 \mathrm{~N} 1$ virus in the French general population. PLoS One 2013;8:e60127.

10 Liao Q, Cowling BJ, Lam WWT, et al. Factors affecting intention to receive and self-reported receipt of 2009 pandemic (H1N1) vaccine in Hong Kong: a longitudinal study. PLoS One 2011;6:e17713.

11 Lin Y, Huang L, Nie S, et al. Knowledge, attitudes and practices (KAP) related to the pandemic (H1N1) 2009 among Chinese general population: a telephone survey. BMC Infect Dis 2011;11:128.

12 Gordon SM. Update on 2009 pandemic influenza A (H1N1) virus. Cleve Clin J Med 2009;76:577-82.

13 Lofgren E, Fefferman NH, Naumov YN, et al. Influenza seasonality: underlying causes and modeling theories. J Virol 2007:81:5429-36.

14 INSEE. Découpage infra-communal en IRIS. http://www.insee.fr/fr/methodes/default. asp?page=zonages/iris.htm (accessed 11 Nov 2013)

15 EMA. European Medicines Agency-Committee for proprietary medicinal products. Note for guidance on harmonization of requirements for influenza vaccines (CPMP) BWP/214/96). 1997. http://www.ema.europa.eu/docs/en_GB/document_library/ Scientific_guideline/2009/09/WC500003945.pdf (accessed 4 Oct 2012).

16 FDA. US Food and Frug Administration-Guidance for Industry: Clinical Data Needed to Support the Licensure of Seasonal Inactivated Influenza Vaccines. 2009. http:// www.fda.gov/BiologicsBloodVaccines/GuidanceComplianceRegulatorylnformation/ Guidances/Vaccines/ucm074794.htm (accessed 4 Oct 2012).

17 Carrat F, Flahault A, Boussard E, et al. Surveillance of influenza-like illness in France. The example of the 1995/1996 epidemic. J Epidemiol Community Health 1998;52(Suppl 1):32S-8S.

18 Byrne BM. Structural equation modeling with Mplus: basic concepts, applications, and programming. Routledge Academic New York, 2011

19 Kieffer $A$, Paboriboune $P$, Crépey $P$, et al. 2009 A(H1N1) seroconversion rates and risk factors among the general population in Vientiane Capital, Laos. PLoS One 2013;8:e61909.

20 Hoschler K, Thompson C, Andrews N, et al. Seroprevalence of influenza A(H1N1) pdm09 virus antibody, England, 2010 and 2011. Emerg Infect Dis 2012;18:1894-7

21 Rubin GJ, Amlôt R, Page L, et al. Public perceptions, anxiety, and behaviour change in relation to the swine flu outbreak: cross sectional telephone survey. BMJ 2009;339:b2651.

22 Andrew MK, McNeil S, Merry $\mathrm{H}$, et al. Rates of influenza vaccination in older adults and factors associated with vaccine use: a secondary analysis of the Canadian Study of Health and Aging. BMC Public Health 2004;4:36.

23 Schwarzinger M, Flicoteaux R, Cortarenoda $S$, et al. Low acceptability of A/H1N1 pandemic vaccination in French adult population: did public health policy fuel public dissonance? PLoS One 2010;5:e10199.

24 Vaux S, Van Cauteren D, Guthmann J-P, et al. Influenza vaccination coverage against seasonal and pandemic influenza and their determinants in France: a cross-sectional survey. BMC Public Health 2011;11:30.

25 Chen MIC, Cook AR, Lim WY, et al. Factors influencing infection by pandemic influenza $A(\mathrm{H} 1 \mathrm{N1}$ )pdm09 over three epidemic waves in Singapore. Influenza Other Respi Viruses 2013;7:1380-9.

26 Cauchemez S, Valleron A-J, Boëlle P-Y, et al. Estimating the impact of school closure on influenza transmission from sentinel data. Nature 2008;452:750-4.

27 Mossong J, Hens N, Jit M, et al. Social contacts and mixing patterns relevant to the spread of infectious diseases. PLoS Med 2008:5:e74

28 Kwok KO, Cowling BJ, Wei VWl, et al. Social contacts and the locations in which they occur as risk factors for influenza infection. Proc Biol Sci 2014;281: 20140709.

29 Kucharski AJ, Kwok KO, Wei VWI, et al. The contribution of social behaviour to the transmission of influenza A in a human population. PLoS Pathog 2014;10:e1004206

30 De $C a o$, Zagheni $E$, Manfredi $P$, et al. The relative importance of frequency of contacts and duration of exposure for the spread of directly transmitted infections. Biostatistics 2014;15:470-83

31 Melegaro A, Jit M, Gay N, et al. What types of contacts are important for the spread of infections?: using contact survey data to explore European mixing patterns. Epidemics 2011;3:143-51.

32 McElhaney JE, Xie D, Hager WD, et al. T cell responses are better correlates of vaccine protection in the elderly. J Immunol 2006;176:6333-9.

33 Franco LM, Bucasas KL, Wells JM, et al. Integrative genomic analysis of the human immune response to influenza vaccination. Elife 2013;2:e00299-e00299.

34 DiLalla LF. A structural equation modeling overview for medical researchers. J Dev Behav Pediatr 2008;29:51-4. 